\title{
Approximate Solution of Fuzzy Matrix Equations with LR Fuzzy Numbers
}

\author{
Xiaobin Guo ${ }^{1}$, Dequan Shang ${ }^{2}$ \\ ${ }^{1}$ College of Mathematics and Statistics, Northwest Normal University, Lanzhou, China \\ ${ }^{2}$ Department of Public Courses, Gansu College of Chinese Medicine, Lanzhou, China \\ Email: guoxb@nwnu.edu.cn,gxbglz@163.com
}

Received August 10, 2012; revised September 12, 2012; accepted September 20, 2012

\begin{abstract}
In the paper, a class of fuzzy matrix equations $A \tilde{X}=\tilde{B}$ where $A$ is an $m \times n$ crisp matrix and $\tilde{B}$ is an $m \times p$ arbitrary LR fuzzy numbers matrix, is investigated. We convert the fuzzy matrix equation into two crisp matrix equations. Then the fuzzy approximate solution of the fuzzy matrix equation is obtained by solving two crisp matrix equations. The existence condition of the strong LR fuzzy solution to the fuzzy matrix equation is also discussed. Some examples are given to illustrate the proposed method. Our results enrich the fuzzy linear systems theory.
\end{abstract}

Keywords: LR Fuzzy Numbers; Matrix Analysis; Fuzzy Matrix Equations; Fuzzy Approximate Solution

\section{Introduction}

Systems of simultaneous matrix equations are essential mathematical tools in science and technology. In many applications, at least some of the parameters of the system are represented by fuzzy rather than crisp numbers. So, it is very important to develop a numerical procedure that would appropriately handle and solve fuzzy matrix systems. The concept of fuzzy numbers and arithmetic operations were first introduced and investigated by Zadeh [1] and Dubois [2].

Since M. Friedman et al. [3] proposed a general model for solving a $n \times n$ fuzzy linear systems whose coefficients matrix is crisp and the right-hand side is a fuzzy number vector in 1998, many works have been done about how to deal with some advanced fuzzy linear systems such as dual fuzzy linear systems (DFLS), general fuzzy linear systems (GFLS), fully fuzzy linear systems (FFLS), dual fully fuzzy linear systems (DFFLS) and general dual fuzzy linear systems (GDFLS), see [4-9]. However, for a fuzzy linear matrix equation which always has a wide use in control theory and control engineering, few works have been done in the past decades. In 2010, Gong Zt $[10,11]$ investigated a class of fuzzy matrix equations $A \tilde{X}=\tilde{B}$ by means of the undetermined coefficients method, and studied least squares solutions of the inconsistent fuzzy matrix equation by using generalized inverses. In 2011, Guo X. B. [12] studied the minimal fuzzy solution of fuzzy Sylvester matrix equations $A \tilde{X}+\tilde{X} B=\tilde{C}$. Recently, they [13] considered the fuzzy symmetric solutions of fuzzy matrix equations

$$
A \tilde{X}=\tilde{B} .
$$

The LR fuzzy number and its operations were firstly introduced by Dubois [2]. In 2006, Dehgham et al. [6] discussed the computational methods for fully fuzzy linear systems whose coefficient matrix and the right-hand side vector are denoted by LR fuzzy numbers. In this paper, we propose a practical method for solving a class of fuzzy matrix system $A \tilde{X}=\tilde{B}$ in which $A$ is an $m \times n$ crisp matrix and $\tilde{B}$ is an $m \times p$ arbitrary LR fuzzy numbers matrix. In contrast, the contribution of this paper is to generalize Dubois' definition and arithmetic operation of LR fuzzy numbers and then use this result to solve fuzzy matrix systems numerically. The importance of converting fuzzy linear system into two systems of linear equations is that any numerical approach suitable for system of linear equations may be implemented. In addition, since our model does not contain parameter $r$, $0 \leq r \leq 1$, its numerical computation is relatively easy.

\section{Preliminaries}

Definition 2.1. [2] A fuzzy number $\tilde{M}$ is said to be a LR fuzzy number if

$$
\mu_{\tilde{M}}(x)=\left\{\begin{array}{l}
L\left(\frac{m-x}{\alpha}\right), x \leq m, \alpha>0, \\
R\left(\frac{x-m}{\beta}\right), x \geq m, \beta>0,
\end{array}\right.
$$

where $m$ is the mean value of $\tilde{M}$, and $\alpha$ and $\beta$ are left and right spreads, respectively. The function $L($.), 
which is called left shape function satisfying: 1$) L(x)=$ $L(-x)$; 2) $L(0)=1$ and $L(1)=0$; 3) $L(x)$ is a non increasing on $[0,+\infty)$.

The definition of a right shape function $L($.$) is usually$ similar to that of $L($.$) . A LR fuzzy number \tilde{M}$ is symbolically shown as $M=(m, \alpha, \beta)_{L R}$.

Noticing that $\alpha>0, \quad \beta>0$ in Definition 2.1, which limits its applications, we extend the definition of LR fuzzy numbers as follows.

Definition 2.2. (Generalized LR fuzzy numbers) Let $\tilde{M}=(m, \alpha, \beta)_{L R}$, we define

1) if $\alpha<0$ and $\beta>0$, then $\tilde{M}=(m, 0, \operatorname{Max}(-\alpha, \beta))_{L R}$, and

$$
\mu_{\tilde{M}}(x)=\left\{\begin{array}{cc}
0, & x \leq m, \\
R\left(\frac{x-m}{\max (-\alpha, \beta)}\right), & x \geq m .
\end{array}\right.
$$

2) if $\alpha>0$ and $\beta<0$, then

$\tilde{M}=(m, \operatorname{Max}(-\alpha, \beta), 0)_{L R}$, and

$$
\mu_{\tilde{M}}(x)=\left\{\begin{array}{cc}
L\left(\frac{m-x}{\max \{\alpha,-\beta\}}\right), & x \leq m, \\
0, & x \geq m .
\end{array}\right.
$$

3) if $\alpha<0$ and $\beta<0$, then $\tilde{M}=(m,-\beta,-\alpha)_{L R}$, and

$$
\mu_{\tilde{M}}(x)= \begin{cases}L\left(\frac{m-x}{-\beta}\right), & x \leq m, \\ R\left(\frac{x-m}{-\alpha}\right), & x \geq m .\end{cases}
$$

For arbitrary LR fuzzy number $\tilde{M}=(m, \alpha, \beta)_{L R}$ and $\tilde{N}=(m, \gamma, \delta)_{L R}$, we have

1) $\tilde{M}+\tilde{N}=(m+n, \alpha+\gamma, \beta+\delta)_{L R}$.

2) $\lambda \tilde{N}= \begin{cases}(\lambda n, \lambda \gamma, \lambda \delta)_{L R}, & \lambda \geq 0 \\ (\lambda n,-\lambda \delta,-\lambda \gamma)_{R L}, & \lambda<0 .\end{cases}$

Definition 2.3. The matrix system

$$
\begin{aligned}
& \left(\begin{array}{cccc}
a_{11} & a_{12} & \cdots & a_{1 n} \\
a_{21} & a_{22} & \cdots & a_{2 n} \\
\cdots & \cdots & \cdots & \cdots \\
a_{m 1} & a_{m 2} & \cdots & a_{m n}
\end{array}\right)\left(\begin{array}{llll}
\tilde{x}_{11} & \tilde{x}_{12} & \cdots & \tilde{x}_{1 p} \\
\tilde{x}_{21} & \tilde{x}_{22} & \cdots & \tilde{x}_{2 p} \\
\cdots & \cdots & \cdots & \cdots \\
\tilde{x}_{n 1} & \tilde{x}_{n 2} & \cdots & \tilde{x}_{n p}
\end{array}\right) \\
& =\left(\begin{array}{cccc}
\tilde{b}_{11} & \tilde{b}_{12} & \cdots & \tilde{b}_{1 p} \\
\tilde{b}_{21} & \tilde{b}_{22} & \cdots & \tilde{b}_{2 p} \\
\cdots & \cdots & \cdots & \cdots \\
\tilde{b}_{m 1} & \tilde{b}_{m 2} & \cdots & \tilde{b}_{m p}
\end{array}\right)
\end{aligned}
$$

where $a_{i j}$ are crisp numbers and $\tilde{b}_{i j}$ are LR fuzzy numbers, is called a LR fuzzy matrix equation (LRFME).

Using matrix notation, we have

$$
A \tilde{X}=\tilde{B} .
$$

A LR fuzzy numbers matrix

$$
\begin{gathered}
\tilde{X}=\left(\tilde{x}_{i j}\right)_{n \times p}^{T}, \quad \tilde{x}_{i j}=\left(x_{i j}, x_{i j}^{l}, x_{i j}^{r}\right)_{L R}, \\
1 \leq i \leq n, \quad 1 \leq j \leq p
\end{gathered}
$$

is called a solution of the LR fuzzy matrix systems if $\tilde{X}$ satisfies (2).

\section{Method for Solving LRFME}

In this section we investigate the LR fuzzy matrix system (2). Firstly, we propose a model for solving the LR fuzzy matrix system, i.e., convert it into two crisp systems of matrix equations. Then we define the LR fuzzy solution and give its solution representation to the original fuzzy matrix system. At last, the existence condition of the strong LR fuzzy solution to the original fuzzy matrix system is also discussed.

\subsection{Extended Crisp Matrix Equations}

By using arithmetic operations of LR fuzzy numbers, we extend the LR fuzzy matrix Equation (2) into two crisp matrix equations.

Theorem 3.1. The LR dual fuzzy linear Equation (2) can be extended into two crisp systems of linear equations as follows:

$$
A X=B,
$$

i.e.,

$$
\begin{aligned}
& \left(\begin{array}{cccc}
a_{11} & a_{12} & \cdots & a_{1 n} \\
a_{21} & a_{22} & \cdots & a_{2 n} \\
\cdots & \cdots & \cdots & \cdots \\
a_{m 1} & a_{m 2} & \cdots & a_{m n}
\end{array}\right)\left(\begin{array}{cccc}
x_{11} & x_{12} & \cdots & x_{1 p} \\
x_{21} & x_{22} & \cdots & x_{2 p} \\
\cdots & \cdots & \cdots & \cdots \\
x_{n 1} & x_{n 2} & \cdots & x_{n p}
\end{array}\right) \\
& =\left(\begin{array}{cccc}
b_{11} & b_{12} & \cdots & b_{1 p} \\
b_{21} & b_{22} & \cdots & b_{2 p} \\
\cdots & \cdots & \cdots & \cdots \\
b_{m 1} & b_{m 2} & \cdots & b_{m p}
\end{array}\right)
\end{aligned}
$$

and

$$
S\left(\begin{array}{c}
X^{l} \\
X^{r}
\end{array}\right)=\left(\begin{array}{c}
B^{l} \\
B^{r}
\end{array}\right)=F,
$$




$$
\left(\begin{array}{cccc}
s_{11} & s_{12} & \cdots & s_{1,2 n} \\
s_{21} & s_{22} & \cdots & s_{2,2 n} \\
\cdots & \cdots & \cdots & \cdots \\
s_{2 m, 1} & S_{2 m, 2} & \cdots & s_{2 m, 2 n}
\end{array}\right)\left(\begin{array}{cccc}
x_{11}^{l} & x_{12}^{l} & \cdots & x_{1 p}^{l} \\
x_{21}^{l} & x_{22}^{l} & \cdots & x_{2 p}^{l} \\
\cdots & \cdots & \cdots & \cdots \\
x_{n 1}^{r} & x_{n 2}^{r} & \cdots & x_{n p}^{r} \\
x_{11}^{r} & x_{12}^{r} & \cdots & x_{1 p}^{r} \\
x_{21}^{r} & x_{22}^{r} & \cdots & x_{2 p}^{r} \\
\cdots & \cdots & \cdots & \cdots \\
x_{n 1}^{r} & x_{n 1}^{r} & \cdots & x_{n p}^{r}
\end{array}\right)=\left(\begin{array}{cccc}
b_{11}^{l} & b_{12}^{l} & \cdots & b_{1 p}^{l} \\
b_{21}^{l} & b_{22}^{l} & \cdots & b_{2 p}^{l} \\
\cdots & \cdots & \cdots & \cdots \\
b_{m 1}^{l} & b_{m 1}^{l} & \cdots & b_{m p}^{l} \\
b_{11}^{r} & b_{12}^{r} & \cdots & b_{1 p}^{r} \\
b_{21}^{r} & b_{22}^{r} & \cdots & b_{2 p}^{r} \\
\cdots & \cdots & \cdots & \cdots \\
b_{m 1}^{r} & b_{m 1}^{r} & \cdots & b_{m p}^{r}
\end{array}\right),
$$

where $s_{i j}, 1 \leq i \leq 2 m, 1 \leq j \leq 2 n$ are determined as follows:

If $a_{i j} \geq 0$, then $s_{i j}=a_{i j}, s_{m+i, n+j}=a_{i j}$; if $a_{i j}<0$, then $s_{i, n+j}=a_{i j}, s_{m+i, n}=a_{i j}$, and any $s_{k l}$ which is not determined by the above items is zero, $1 \leq k \leq 2 m$, $1 \leq l \leq 2 n$.

Proof. Let $\tilde{X}=\left(\tilde{X}_{1}, \tilde{X}_{2} \cdots, \tilde{X}_{p}\right)$,

$$
\tilde{X}_{j}=\left(\left(x_{1 j}, x_{1 j}^{l}, x_{1 j}^{r}\right)_{L R}, \cdots,\left(x_{n j}, x_{n j}^{l}, x_{n j}^{r}\right)_{L R}\right)^{T}
$$

and $\tilde{B}=\left(\tilde{B}_{1}, \tilde{B}_{2} \cdots, \tilde{B}_{p}\right)$,

$$
\tilde{B}_{j}=\left(\left(b_{1 j}, b_{1 j}^{l}, b_{1 j}^{r}\right)_{L R}, \cdots,\left(b_{n j}, b_{n j}^{l}, b_{n j}^{r}\right)_{L R}\right)^{T} .
$$

Then the fuzzy matrix Equation (1) can be rewritten in the block forms

$$
A\left(\tilde{X}_{1}, \tilde{X}_{2} \cdots, \tilde{X}_{p}\right)=\left(\tilde{B}_{1}, \tilde{B}_{2} \cdots, \tilde{B}_{p}\right),
$$

Thus the original system (1) is equivalent to the following fuzzy linear equations

$$
A \tilde{X}_{j}=\tilde{B}_{j}, 1 \leq j \leq p .
$$

Now we consider the Equations (4). Let $a_{i}$ be the $i$ th row of matrix $A, 1 \leq i \leq m$, we can represent $\left[A \tilde{X}_{j}\right]_{i}$ in the form $\left[A \tilde{X}_{j}\right]_{i}=a_{i} \tilde{X}_{j}, \quad i=1,2, \cdots, m$.

Denoting $Q_{i}^{+}=\left\{a_{i k}: a_{i k} \geq 0\right\}$ and $Q_{i}^{-}=\left\{a_{i k}: a_{i k}<0\right\}$, we have

$$
\left[A \tilde{X}_{j}\right]_{i}=\sum_{k \in Q_{j}^{+}} a_{i k} \tilde{x}_{k j}+\sum_{k \in Q_{j}^{-}} a_{i k} \tilde{x}_{k j}, i=1,2, \cdots, m .
$$

i.e.,

$$
\begin{aligned}
{\left[A \tilde{X}_{j}\right]_{i}=} & \left(\sum_{k \in Q_{j}^{+}} a_{i k} x_{k j}-\sum_{k \in Q_{j}^{-}} a_{i k} x_{k j}, \sum_{k \in Q_{j}^{+}} a_{i k} x_{k j}^{l}\right. \\
& \left.-\sum_{k \in Q_{j}^{-}} a_{i k} x_{k j}^{r}, \sum_{k \in Q_{j}^{+}} a_{i k} x_{k j}^{r}-\sum_{k \in Q_{j}^{+}} a_{i k} x_{k j}^{l}\right)_{L R}
\end{aligned}
$$

Consider the given LR fuzzy vector

$$
\tilde{B}_{j}=\left(\left(b_{1 j}, b_{1 j}^{l}, b_{1 j}^{r}\right)_{L R}, \cdots,\left(b_{n j}, b_{n j}^{l}, b_{n j}^{r}\right)_{L R}\right)^{T},
$$

we can write the system (2) as

$$
\begin{aligned}
& \left(\sum_{k \in Q_{j}^{+}} a_{i k} x_{k j}-\sum_{k \in Q_{j}^{-}} a_{i k} x_{k j}, \sum_{k \in Q_{j}^{+}} a_{i k} x_{k j}^{l}-\sum_{k \in Q_{j}^{-}} a_{i k} x_{k j}^{r},\right. \\
& \left.\sum_{k \in Q_{j}^{+}} a_{i k} x_{k j}^{r}-\sum_{k \in Q_{j}^{+}} a_{i k} x_{k j}^{l}\right)_{L R}=\left(B_{j}, B_{j}^{l}, B_{j}^{r}\right)_{L R}
\end{aligned}
$$

Suppose the system $A \tilde{X}_{j}=\tilde{B}_{j}, \quad 1 \leq j \leq p$ has a solution. Then, the corresponding mean value $X_{j}=\left(x_{1 j}, x_{2 j}, \cdots, x_{n j}\right)^{T}$ of the solution must lie in the following linear system

$$
\left(\begin{array}{cccc}
a_{11} & a_{12} & \cdots & a_{1 n} \\
a_{21} & a_{22} & \cdots & a_{2 n} \\
\cdots & \cdots & \cdots & \cdots \\
a_{m 1} & a_{m 2} & \cdots & a_{m n}
\end{array}\right)\left(\begin{array}{c}
x_{1 j} \\
x_{2 j} \\
\cdots \\
x_{n j}
\end{array}\right)=\left(\begin{array}{c}
b_{1 j} \\
b_{2 j} \\
\cdots \\
b_{n j}
\end{array}\right) .
$$

Meanwhile, the left spread $X_{j}{ }^{l}=\left(x_{1 j}^{l}, x_{2 j}^{l}, \cdots, x_{n j}^{l}\right)^{T}$ and the right spread $X_{j}^{r}=\left(x_{1 j}^{r}, x_{2 j}^{r}, \cdots, x_{n j}^{r}\right)^{T}$ of the solution can be derived from solving the following crisp linear system

$$
\left(\begin{array}{cccc}
s_{11} & s_{12} & \cdots & s_{1,2 n} \\
s_{21} & s_{22} & \cdots & s_{2,2 n} \\
\cdots & \cdots & \cdots & \cdots \\
s_{2 m, 1} & s_{2 m, 2} & \cdots & s_{2 m, 2 n}
\end{array}\right)\left(\begin{array}{c}
x_{1 j}^{l} \\
\vdots \\
x_{n j}^{l} \\
x_{1 j}^{r} \\
\vdots \\
x_{n j}^{r}
\end{array}\right)=\left(\begin{array}{c}
b_{1 j}^{l} \\
\vdots \\
b_{n j}^{l} \\
b_{1 j}^{r} \\
\vdots \\
b_{n j}^{r}
\end{array}\right) .
$$

Finally, we restore the Equation (5) and obtain above matrix Equations (3) and (4).

The proof is completed.

\subsection{Computing Model Matrix Equations}

In order to solve the original fuzzy linear Equation (2), 
we need to consider crisp matrix Equations (3) and (4). Since Equations (3) and (4) are crisp, their computation is relatively easy.

In general [14], the minimal solutions of matrix systems (3) and (4) can be expressed uniformly by

$$
X=A^{+} B
$$

and

$$
\left(\begin{array}{l}
X^{l} \\
X^{r}
\end{array}\right)=S^{+}\left(\begin{array}{l}
B^{l} \\
B^{r}
\end{array}\right)=S^{+} F
$$

respectively, no matter the Equations (3) and (4) are consistent or not.

It seems that we have obtained the solution of the original fuzzy linear system (2) as follows:

$$
\begin{aligned}
\tilde{X} & =\left(X, X^{l}, X^{r}\right)_{L R} \\
& =\left(A^{+} d,\left(I_{n} O\right) S^{+} F,\left(O I_{n}\right) S^{+} F\right)_{L R}
\end{aligned}
$$

But the solution vector may still not be an appropriate $L R$ fuzzy numbers vector except for $S^{+} F \geq 0$. So we give the definition of the minimal LR fuzzy solution to the Equation (2) as follows:

Definition 3.1. Let $\tilde{X}=\left(x_{i j}, x_{i j}^{l}, x_{i j}^{r}\right)_{L R}$, $1 \leq i \leq n, 1 \leq j \leq p$. If $X=\left(x_{i j}\right)_{n \times p}$ is the minimal solution of Equation (3), $X^{l}=\left(x_{i j}\right)_{n \times p}$ and $X^{r}=\left(x_{i j}^{r}\right)_{n \times p}$ are minimal solution of Equation (4) such that $X^{l} \geq 0$, $X^{r} \geq 0$, then we call $\tilde{X}=\left(X, X^{l}, X^{r}\right)_{L R}$ is a strong LR fuzzy solution of Equation (2). Otherwise, it is a weak LR fuzzy solution of Equation (2) given by

$$
\begin{gathered}
\tilde{x}_{i j}= \begin{cases}\left(x_{i j}, x_{i j}^{l}, x_{i j}^{r}\right)_{L R}, & x_{i j}^{l}>0, x_{i j}^{r}>0, \\
\left(x_{i j}, 0, \max \left\{-x_{i j}^{l}, x_{i j}^{r}\right\}\right)_{L R}, & x_{i j}^{l}<0, x_{i j}^{r}>0, \\
\left(x_{i j}, \max \left\{x_{i j}^{l},-x_{i j}^{r}\right\}, 0\right)_{L R}, & x_{i j}^{l}>0, x_{i j}^{r}<0, \\
\left(x_{i j},-x_{i j}^{r},-x_{i j}^{l}\right)_{L R}, & x_{i j}^{l}<0, x_{i j}^{r}<0 .\end{cases} \\
1 \leq i \leq n, 1 \leq j \leq p .
\end{gathered}
$$

\subsection{A Sufficient Condition of Strong Fuzzy Solution}

The key points to make the solution vector being a LR fuzzy solution are $X^{l} \geq 0$ and $X^{r} \geq 0$. Since $X^{l}=\left(I_{n} O\right) S^{+} F, X^{r}=\left(O I_{n}\right) S^{+} F$, we know that the non negativities of $X^{l}$ and $X^{r}$ are equivalent to the condition $S^{+} \geq 0$ now that $\left(\begin{array}{l}X^{l} \\ X^{r}\end{array}\right) \geq 0$ is known.
By the above analysis, we have the following result.

Theorem 3.2. Let $A$ belong to $R^{m \times n}$. If $S^{+}$is nonnegative, the solution of the LR fuzzy matrix system (2) is expressed by

$$
\begin{aligned}
\tilde{X} & =\left(X, X^{l}, X^{r}\right)_{L R} \\
& =\left(A^{+} d,\left(I_{n} O\right) S^{+} F,\left(O I_{n}\right) S^{+} F\right)_{L R}
\end{aligned}
$$

and it admits a strong minimal LR fuzzy solution.

The following Theorem gives a result for such $S^{+}$to be nonnegative.

Theorem 3.3. [15] Let $S$ be an $2 p \times 2 p$ nonnegative matrix with rank $r$. Then the following assertions are equivalent:

1) $S^{+} \geq 0$;

2) There exists a permutation matrix $P$, such that $P S$ has the form

$$
P S=\left(\begin{array}{c}
Q_{1} \\
\vdots \\
Q_{r} \\
O
\end{array}\right),
$$

where each $Q_{i}$ has rank 1 and the rows of $Q_{i}$ are orthogonal to the rows of $Q_{i}$, whenever $i \neq j$, the zero matrix may be absent.

$$
\text { 3) } S^{+}=\left(\begin{array}{ll}
H E^{T} & H F^{T} \\
H F^{T} & H E^{T}
\end{array}\right)
$$

for some positive diagonal matrix $H$. In this case,

$$
(E+F)^{+}=H(E+F)^{T},(E-F)^{+}=H(E-F)^{T} .
$$

\section{Numerical Examples}

In this section, we work out two numerical examples to illustrate the proposed method.

Example 4.1. Consider the fuzzy matrix systems:

$$
\left(\begin{array}{ccc}
1 & 0 & -1 \\
1 & -1 & 0 \\
2 & 1 & 1
\end{array}\right)\left(\begin{array}{cc}
\tilde{x}_{11} & \tilde{x}_{12} \\
\tilde{x}_{21} & \tilde{x}_{22} \\
\tilde{x}_{31} & \tilde{x}_{32}
\end{array}\right)=\left(\begin{array}{ll}
(2,1,1)_{L R} & (3,2,1)_{L R} \\
(2,1,2)_{L R} & (2,1,2)_{L R} \\
(6,3,2)_{L R} & (5,2,3)_{L R}
\end{array}\right) .
$$

The coefficient matrix $A$ is nonsingular and the extended matrix $S$ is singular. By the Theorem 3.1., the mean value $x$, the left spread $x^{l}$ and the right spread $x^{r}$ of solution are obtained from

$$
\left(\begin{array}{ccc}
1 & 0 & -1 \\
1 & -1 & 0 \\
2 & 1 & 1
\end{array}\right)\left(\begin{array}{ll}
x_{11} & x_{12} \\
x_{21} & x_{22} \\
x_{31} & x_{32}
\end{array}\right)=\left(\begin{array}{ll}
2 & 3 \\
2 & 2 \\
6 & 5
\end{array}\right)
$$

and 


$$
\left(\begin{array}{llllll}
1 & 1 & 0 & 0 & 0 & 1 \\
1 & 0 & 0 & 0 & 1 & 0 \\
2 & 1 & 1 & 0 & 0 & 0 \\
0 & 0 & 1 & 1 & 1 & 0 \\
0 & 1 & 0 & 1 & 0 & 0 \\
0 & 0 & 0 & 2 & 1 & 1
\end{array}\right)\left(\begin{array}{ll}
x_{11}^{l} & x_{12}^{l} \\
x_{21}^{l} & x_{22}^{l} \\
x_{31}^{l} & x_{32}^{l} \\
x_{11}^{r} & x_{12}^{r} \\
x_{21}^{r} & x_{22}^{r} \\
x_{31}^{r} & x_{32}^{r}
\end{array}\right)=\left(\begin{array}{ll}
1 & 2 \\
1 & 1 \\
3 & 2 \\
1 & 1 \\
2 & 2 \\
2 & 3
\end{array}\right) .
$$

Thus, we have

$$
\begin{aligned}
& x=A^{+} B=\left(\begin{array}{ll}
2.5000, & 2.5000 \\
0.5000, & 0.5000 \\
0.5000, & -0.500
\end{array}\right) \\
& \tilde{X}=\left(\begin{array}{ll}
\tilde{X}_{11} & \tilde{X}_{12} \\
\tilde{X}_{21} & \tilde{X}_{22} \\
\tilde{X}_{31} & \tilde{X}_{32}
\end{array}\right)=\left(\begin{array}{llllll}
(2.5000, & 0.8333, & 0.8333)_{L R} & (2.5000, & 0.7639, & 1.0139)_{L R} \\
(0.5000, & 1.1667, & 0.1667)_{L R} & (0.5000, & 0.8194, & 0.0694)_{L R} \\
(0.5000, & 0.1667, & 0.1667)_{L R} & (-0.500, & 0.0000, & 1.0694)_{L R}
\end{array}\right)
\end{aligned}
$$

Since $x_{23}^{l}$ is negative, according to Definition 3.1., the LR fuzzy approximate solution of the original fuzzy

and it admits a strong LR fuzzy solution.

Example 4.2. Consider the following matrix systems:

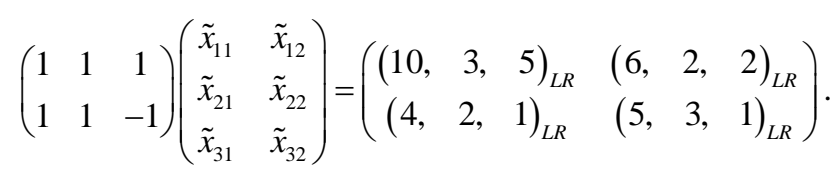

By the Theorem 3.1., the mean value $x$ of solution lies in the following crisp matrix system

$$
\left(\begin{array}{ccc}
1 & 1 & 1 \\
1 & 1 & -1
\end{array}\right)\left(\begin{array}{ll}
x_{11} & x_{12} \\
x_{21} & x_{22} \\
x_{31} & x_{32}
\end{array}\right)=\left(\begin{array}{cc}
10 & 6 \\
4 & 5
\end{array}\right)
$$

Meanwhile, the left spread $x^{l}$ and the right spread $x^{r}$ of solution are obtained by solving the following crisp matrix system

$$
\begin{aligned}
& \left(\begin{array}{llllll}
1 & 1 & 1 & 0 & 0 & 0 \\
1 & 1 & 0 & 0 & 0 & 1 \\
0 & 0 & 0 & 1 & 1 & 1 \\
0 & 0 & 1 & 1 & 1 & 0
\end{array}\right)\left(\begin{array}{ll}
x_{11}^{l} & x_{12}^{l} \\
x_{21}^{l} & x_{22}^{l} \\
x_{31}^{l} & x_{32}^{l} \\
x_{11}^{r} & x_{12}^{r} \\
x_{21}^{r} & x_{22}^{r} \\
x_{31}^{r} & x_{32}^{r}
\end{array}\right)=\left(\begin{array}{ll}
3 & 2 \\
2 & 3 \\
5 & 2 \\
1 & 1
\end{array}\right) . \\
& \qquad \tilde{X}=\left(\begin{array}{ll}
\tilde{x}_{11} & \tilde{x}_{12} \\
\tilde{x}_{21} & \tilde{x}_{22} \\
\tilde{x}_{31} & \tilde{x}_{32}
\end{array}\right)=\left(\begin{array}{ll}
(3.50, & 0.792, \\
(3.50, & 0.792, \\
(3.00, & 0.167,
\end{array}\right. \\
& \text { 5. Conclusion } \\
& \text { In this work we proposed a general model for solving the } \\
& \text { fuzzy matrix equation } A \tilde{X}=\tilde{B} \text { where } A \text { is an } m \times n \\
& \text { crisp matrix and } \tilde{B} \text { is a } n \times p \text { arbitrary LR fuzzy }
\end{aligned}
$$$$
\tilde{X}=\left(\begin{array}{ll}
\tilde{x}_{11} & \tilde{x}_{12} \\
\tilde{x}_{21} & \tilde{x}_{22} \\
\tilde{x}_{31} & \tilde{x}_{32}
\end{array}\right)=\left(\begin{array}{llllll}
(3.50, & 0.792, & 1.042)_{L R} & (2.75, & 0.917, & 0.417)_{L R} \\
(3.50, & 0.792, & 1.042)_{L R} & (2.75, & 0.917, & 0.417)_{L R} \\
(3.00, & 0.167, & 1.667)_{L R} & (0.50, & 0.167, & 1.167)_{L R}
\end{array}\right) .
$$
strong LR fuzzy approximate solution given by

\section{Conclusion}

By Definition 3.1., the original fuzzy system has a

Thus, we have

$$
x=A^{+} B=\left(\begin{array}{ll}
3.5000, & 2.7500 \\
3.5000, & 2.7500 \\
3.0000, & 0.5000
\end{array}\right)
$$

and

$$
\left(\begin{array}{l}
x^{l} \\
x^{r}
\end{array}\right)=S^{+}\left(\begin{array}{l}
B^{l} \\
B^{r}
\end{array}\right)=\left(\begin{array}{ll}
0.7917, & 0.9167 \\
0.7917, & 0.9167 \\
0.1667, & 0.1667 \\
1.0417, & 0.4167 \\
1.0417, & 0.4167 \\
1.1667, & 1.1667
\end{array}\right)>0
$$

numbers matrix. We converted the fuzzy matrix system into two crisp matrix equations and obtained the LR fuzzy solution to the original fuzzy system by solving crisp matrix equations. Moreover, the existence condition of strong LR fuzzy solution was studied. Numerical ex- 
amples showed that our method is effective to solve LR fuzzy matrix equations.

\section{Acknowledgements}

The authors are very thankful for the reviewer's helpful suggestions to improve the paper. This research was financially supported by the National Natural Science Foundation of China (Grant No. 71061013) and the Youth Scientific Research Ability Promotion Project of Northwest Normal University (NWNU-LKQN-11-20).

\section{REFERENCES}

[1] L A. Zadeh, "Fuzzy Sets," Information and Control, Vol. 8, No. 3, 1965, pp. 338-353. doi:10.1016/S0019-9958(65)90241-X

[2] D. Dubois, and H. Prade, "Operations on Fuzzy Numbers," International Journal of Systems Science, Vol. 9, No. 3, 1978, pp. 613-626. doi:10.1080/00207727808941724

[3] M. Friedman, M. Ma and A. Kandel, "Fuzzy Linear Systems,” Fuzzy Sets and Systems, Vol. 96, No. 2, 1998, pp. 201-209. doi:10.1016/S0165-0114(96)00270-9

[4] M. Friedman, M. Ma and A. Kandel, "Duality in Fuzzy Linear Systems,” Fuzzy Sets and Systems, Vol. 109, No. 1, 2000, pp. 55-58. doi:10.1016/S0165-0114(98)00102-X

[5] T. Allahviranloo, "Numerical Methods for Fuzzy System of Linear Equations," Applied Mathematics and Computation, Vol. 155, No. 2, 2004, pp. 493-502. doi:10.1016/S0096-3003(03)00793-8

[6] B. Asady, S. Abbasbandy and M. Alavi, "Fuzzy General Linear Systems," Applied Mathematics and Computation, Vol. 169, No. 1, 2005, pp. 34-40. doi:10.1016/j.amc.2004.10.042

[7] K. Wang and B. Zheng, "Inconsistent Fuzzy Linear Systems," Applied Mathematics and Computation, Vol. 181, No. 2, 2006, pp. 973-981. doi:10.1016/j.amc.2006.02.019

[8] M. Dehghan, B. Hashemi and M. Ghatee, "Solution of the Full Fuzzy Linear Systems Using Iterative Techniques," Chaos, Solitons \& Fractals, Vol. 34, No. 2, 2007, pp. 316336. doi:10.1016/j.chaos.2006.03.085

[9] S. Abbasbandy, M. Otadi and M. Mosleh, "Minimal Solution of General Dual Fuzzy Linear Systems," Chaos, Solitons \& Fractals, Vol. 37, No. 4, 2008, pp. 638-652. doi:10.1016/j.chaos.2006.10.045

[10] X. B. Guo and Z. T. Gong, "Undetermined Coefficient Method for Solving Semi-Fuzzy Matrix Equation,” Proceedings of 9th International Conference on Machine Learning and Cybernetics, Qingdao, 11-14 July 2010, pp. 596-600.

[11] Z. T. Gong and X. B. Guo, "Inconsistent Fuzzy Matrix Equations and Its Fuzzy Least Squares Solutions,” Applied Mathematical Modelling, Vol. 35, No. 1, 2011, pp. 1456-1469. doi:10.1016/j.apm.2010.09.022

[12] X. B. Guo, "Approximate Solution of Fuzzy Sylvester Matrix Equations," The 7th International Conference on Computational Intelligence and Security, Sanya, 3-4 December 2011, pp. 52-57.

[13] X. B. Guo and D. Q. Shang, "Fuzzy Symmetric Solutions of Fuzzy Matrix Equations," Advances in Fuzzy Systems, 2012, Article ID: 318069. doi:10.1155/2012/318069

[14] X. D. Zhang, "Matrix Analysis and Its Applications," Tsinghua University and Springer Press, Beijing, 2004.

[15] A. Berman and R. J. Plemmons, "Nonnegative Matrices in the Mathematical Sciences," Academic Press, New York, 1979. 